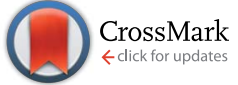

Cite this: RSC Adv., 2017, 7, 180
Received 9th October 2016 Accepted 8th November 2016

DOI: $10.1039 / c 6 r a 24977 a$

www.rsc.org/advances

\title{
Biosynthesis of Ag/almond shell nanocomposite as a cost-effective and efficient catalyst for degradation of 4-nitrophenol and organic dyes
}

\begin{abstract}
Maryam Bordbar*ab
Silver nanoparticles have been immobilized on the surface of waste almond shell using Ruta graveolens sleeves extract as a reducing and stabilizing agent in the present work. FT-IR spectroscopy, photoluminescence spectroscopy, X-ray diffraction (XRD) patterns, field emission scanning electron microscopy (FE-SEM), energy dispersive X-ray spectroscopy (EDS) and transmission electron microscopy (TEM) were used to characterize the almond shell supported silver nanoparticles. The diameters of AgNPs on the almond shell were mostly in the $5-15 \mathrm{~nm}$ range. The catalytic performance of the synthesized catalysts was studied in reduction of 4-nitrophenol (4-NP), rhodamine B (RhB) and methylene blue (MB) at ambient temperature. AgNPs/almond shell was found to be a highly active and recyclable catalyst in the related reaction, which can be recovered and reused seven times with no appreciable loss of catalytic activity.
\end{abstract}

\section{Introduction}

Noble metal nanoparticles (NPs) have been intensively investigated for the past two decades for a wide range of applications including optics, electronics, sensing, catalysis and biomedicine, given their unique and tunable properties. ${ }^{1,2}$ Silver, among the noble metals, is particularly attractive due to its very high electrical conductivity, anti-bacterial activity and relatively low cost. Silver nanoparticles (AgNPs) can be applied as catalysts in a number of important chemical processes, including selective alkane and alkene oxidation, ${ }^{2}$ hydrogenation of dyes, ${ }^{3}$ 4-nitrophenol (4-NP) reduction to 4-aminophenol (4-AP), as an intermediate step in the synthesis of pharmaceutical compounds (e.g. , paracetamol). ${ }^{4}$ As catalysts, AgNPs are usually immobilized onto such appropriate substrates as silica, ${ }^{5}$ alumina, ${ }^{6}$ zeolites, ${ }^{7}$ $\mathrm{TiO}_{2}{ }^{8}$ and different types of carbon compounds ${ }^{9,10}$ so as to (i) prevent their agglomeration and thus the reduction of their catalytic activity, and (ii) increase their efficiency via suitable interaction with the substrate. ${ }^{2}$ There are several green synthetic methods, on the other hand, for obtaining stable and size controlled AgNPs and prevention of their agglomeration. ${ }^{11-13}$ Synthesis of plant mediated silver nanoparticles has been reviewed by Ahmed et al. ${ }^{14}$ Some plant extracts, such as Capsicum annuum L., Jatropha curcas, Argemone mexicana and Citrus limon extracts, have been shown to form stable nanoparticles due to the presence of reducing agents such as

${ }^{a}$ Department of Chemistry, Faculty of Science, University of Qom, Qom 37185-359, Iran. E-mail: m.bordbare@gmail.com; Fax: +98 25 32850953; Tel: +98 2532103792 ${ }^{b}$ Center of Environmental Researches, University of Qom, Qom, Iran alkaloids, polyphenols and flavonoids, which are major phytoconstituents. ${ }^{15-18}$

There are several green synthetic methods for preparation of AgNPs. ${ }^{8,19-21}$ However, the green synthesis of the Ag/almond shell nanocomposite, in which Ruta graveolens leaf extract is used as a reducing agent for $\mathrm{Ag}(\mathrm{I})$ conversion to $\mathrm{Ag}(0)$, has not been reported.

Ruta graveolens (Fig. 1) is an odoriferous herb of Rutaceae family. Ruta graveolens is the Rue or Rue oil source, referred to as Sadab or Satab in Iran. Ruta graveolens has anti-nociceptive, anti-inflammatory and anti-pyretic activities, possibly due to the plant species inhibiting mediators implicated in pain, inflammation and fever. Protocatechuic, vanillic, syringic,

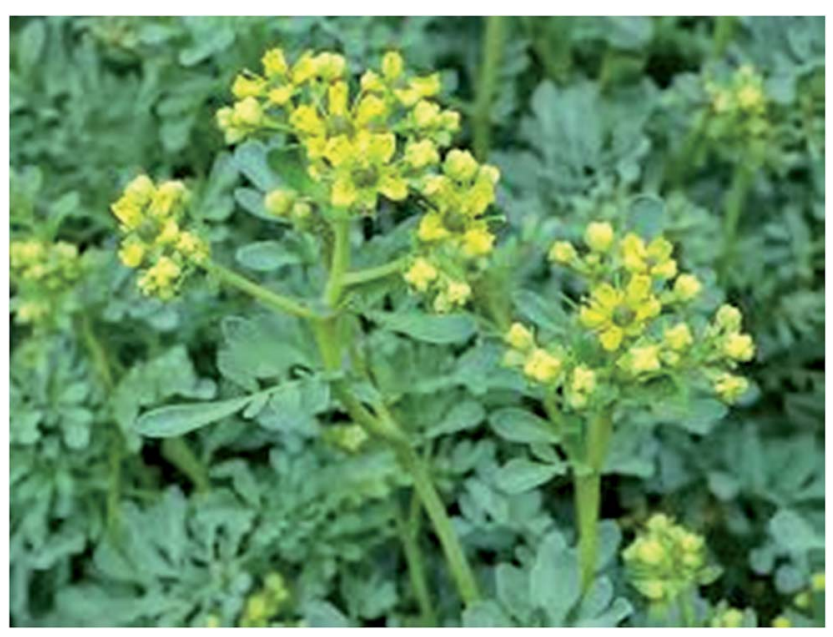

Fig. 1 Image of Ruta graveolens plant. 
<smiles>O=C(O)/C=C/c1ccc(O)cc1</smiles>

p-coumaric acid<smiles>COC1CC(C(=O)O)=CC=C1O</smiles>

Vanillic acid<smiles>COC1CC(C(=O)O)=CC=C1O</smiles>

protocatechuic<smiles>COc1cc(C(=O)O)cc(OC)c1O</smiles>

Syringic acid<smiles>COc1cc2ccc(=O)oc2cc1O</smiles>

scopoletin<smiles>C[C@@H]1[C@@H](COC2O[C@H](C)[C@H](C)[C@@H](C)[C@H]2C)O[C@H](Oc2c(C3=CCC(O)C(O)=C3)oc3cc(O)cc(O)c3c2=O)C[C@H]1C</smiles>

Rutin

Fig. 2 Chemical structures of some compounds in Ruta graveolens extract.

p-coumaric acid and coumarins (Fig. 2) have been detected and quantitatively analyzed in the extract of Ruta graveolens leaves. ${ }^{22}$ Rutin, whose name originates from the rutin containing Ruta graveolens plant, is a flavonoid glycoside of quercetin, found in many plant species. ${ }^{23}$

In this work, cheap agricultural waste almond shells as a naturally valuable resource and environmentally friendly support have been used for immobilization of silver nanoparticles (AgNPs).

A very important crop throughout the world's different temperate regions, almonds (Prunus amygdalus L.) account for $35-75 \%$ of the total fruit weight. ${ }^{24}$ Thus, about $0.8-1.7$ million tons of almond shell is left annually. The ligno-cellulosic material forming the thick endocarp or husk of the almond tree fruit, separated upon processing the fruit to obtain the edible seeds, almond shell is an agricultural waste with no important industrial application, which is usually incinerated or dumped. Air pollution, soil erosion and decreased soil biological activity are caused by burning agricultural wastes. Application of agricultural residues prevents environmental damages and brings about extra income for farmers. ${ }^{25}$ Iran, a major producer of almond, exports thousands of tons of almond annually. Thus, almond shell is easily accessible in the country as an agricultural waste. Almond shell consists of cellulose, hemicelluloses and lignin. ${ }^{24,26}$ The structure of almond shell and its components; especially cellulose, on the other hand, make it appropriate for the production of composites.

The leaf extract of Ruta graveolens has been used in this study as a reducing and stabilizing agent to immobilize AgNPs on the almond shell surface as bio-waste support to form Ag/almond shell nanocomposite in the absence of any harmful reducing or capping agents. This is an environmentally benign alternative route, which avoids the application of hazardous materials. The catalytic activity of Ag/almond shell nanocomposite in the reduction of 4-NP, $\mathrm{RhB}$ and $\mathrm{MB}$ in the presence of $\mathrm{NaBH}_{4}$ in aqueous media has also been studied. Most importantly, only a slight loss is observed in the catalyst activity of $\mathrm{Ag} / \mathrm{almond}$ shell nanocomposite after several recycles.

\section{Experimental}

\subsection{Instruments and reagents}

Highly pure chemicals were purchased from Merck and Aldrich Chemical Companies. FT-IR spectra were recorded on a Varian model 640 spectrophotometer using pressed $\mathrm{KBr}$ pellets. UV-Vis spectra were recorded using a double beam Shimadzu UV-2500 spectrophotometer to ensure the formation of nanoparticles. Varian Cary Eclipse spectrofluorometer was used to record the photoluminescence spectra in a $1 \mathrm{~cm}$ optical path quartz cuvette (scan speed $=100 \mathrm{~nm} \mathrm{m^{-1 }}{ }^{-1}$ slit width $=5 / 5 \mathrm{~nm}$ ). X-ray diffraction (XRD) measurements were carried out using a Philips model X'PertPro diffractometer $(\mathrm{Cu} \mathrm{K} \alpha=1.5406 \AA)$ at a scanning rate of $2^{\circ} \mathrm{min}^{-1}$ in the $2 \theta$ range (10-80). Scanning electron microscopy (SEM) was performed by a TESCAN model VEGA3-XMU equipped with energy dispersive spectroscopy (EDS) analysis. A Philips-EM-2085 transmission electron microscope was used to obtain TEM images at an accelerating voltage of $100.0 \mathrm{kV}$.

\subsection{Preparation of Ruta graveolens leaves extract and Ag/ almond shell nanocomposite}

Dried leaves of Ruta graveolens (10 g) were powdered and refluxed at $80{ }^{\circ} \mathrm{C}$ with deionized water $(60 \mathrm{~mL})$ for $1 \mathrm{~h}$, after which the mixture was allowed to cool to room temperature. The aqueous extract of Ruta graveolens leaves was then centrifuged at $6500 \mathrm{rpm}$ and the supernatant was separated by filtration.

In a typical Ag/almond shell nanocomposite synthesis, first, waste almond shells were washed, dried at $90{ }^{\circ} \mathrm{C}$ for $6 \mathrm{~h}$ in an oven, and mechanically pretreated by crushing in a mill, followed by sieving to separate $<40 \mu \mathrm{m}$ particle size fractions. Then 
the plant extract $(30 \mathrm{~mL})$ was added dropwise to $30 \mathrm{~mL}$ of an $\mathrm{AgNO}_{3}$ solution $(0.3 \mathrm{~g})$ and almond shell powder $(1.0 \mathrm{~g})$ under constant stirring at $70{ }^{\circ} \mathrm{C}$ for $2 \mathrm{~h}$. The resulting mixture was then allowed to cool, filtered, washed with distilled water and dried at $80{ }^{\circ} \mathrm{C}$ in an oven for $12 \mathrm{~h}$.

\subsection{Catalytic reduction of 4-NP, RhB and MB by Ag/almond shell nanocomposite}

In a typical run, the catalyst $(5.0 \mathrm{mg})$ was added to a $2.5 \mathrm{mM}$ 4-NP aqueous solution (25 mL). $0.25 \mathrm{M}$ aqueous solution of freshly prepared $\mathrm{NaBH}_{4}(25 \mathrm{~mL})$ was then added and the mixture obtained was allowed to stir at ambient temperature until the deep yellow color of the solution turned colorless. UV-Vis spectroscopy was used to monitor the reaction progress. Upon completion of the reaction, the catalyst was simply removed from the reaction media by brief centrifugation, washed several times with deionized water and dried for the next cycle.

In addition, the catalyst $(5.0 \mathrm{mg})$ was added to $3.1 \times 10^{-5} \mathrm{M}$ MB aqueous solution ( $25 \mathrm{~mL}) .2 .6 \times 10^{-3} \mathrm{M}$ freshly prepared $\mathrm{NaBH}_{4}$ aqueous solution $(25 \mathrm{~mL})$ was then added and the resulting mixture was allowed to stir at ambient temperature. UV-Vis spectroscopy was used to monitor the reaction progress. Upon completion of the reaction, the catalyst was simply removed from the reaction media by brief centrifugation, washed several times with deionized water and dried for the next cycle. Similar procedures were used in the catalytic reduction of RhB. Equal amounts of $\mathrm{NaBH}_{4}$ and $5.0 \mathrm{mg}$ of the catalyst were used in each catalytic reaction. The concentration of RhB aqueous solution was $2.0 \times 10^{-5} \mathrm{M}$.

\section{Results and discussion}

\subsection{Mechanism of reduction of $\mathrm{AgNO}_{3}$ to $\mathrm{AgNPs}$ by the phytoconstituents of Ruta graveolens}

Ruta graveolens leaf extract has been used as a stabilizing and reducing agent in this work to immobilize AgNPs on the almond shell surface to form Ag/almond shell nanocomposite (Fig. 3). The reduction of $\operatorname{Ag}(\mathrm{I})$ ions and the formation of the corresponding AgNPs may be accounted for by the presence of antioxidant phenolic compound (protocatechuic, vanillic, syringic, $p$-coumaric acid and rutin) in the extract functioning as potent reducing agents.

Fig. 4 shows the proposed mechanism for the reduction of $\mathrm{Ag}^{+}$. In this figure, $\mathrm{Ag}^{+}$ions can form intermediate complexes with phenolic $\mathrm{OH}$ groups, which are subsequently oxidized to quinone, reducing $\mathrm{Ag}^{+}$to AgNPs. ${ }^{15}$

\subsection{Characterization of aqueous extract of Ruta graveolens and AgNPs}

The bands in 300-400 $\mathrm{nm}$ regions in the UV spectrum of leaf extract of the Ruta graveolens (Fig. 5) are associated with the

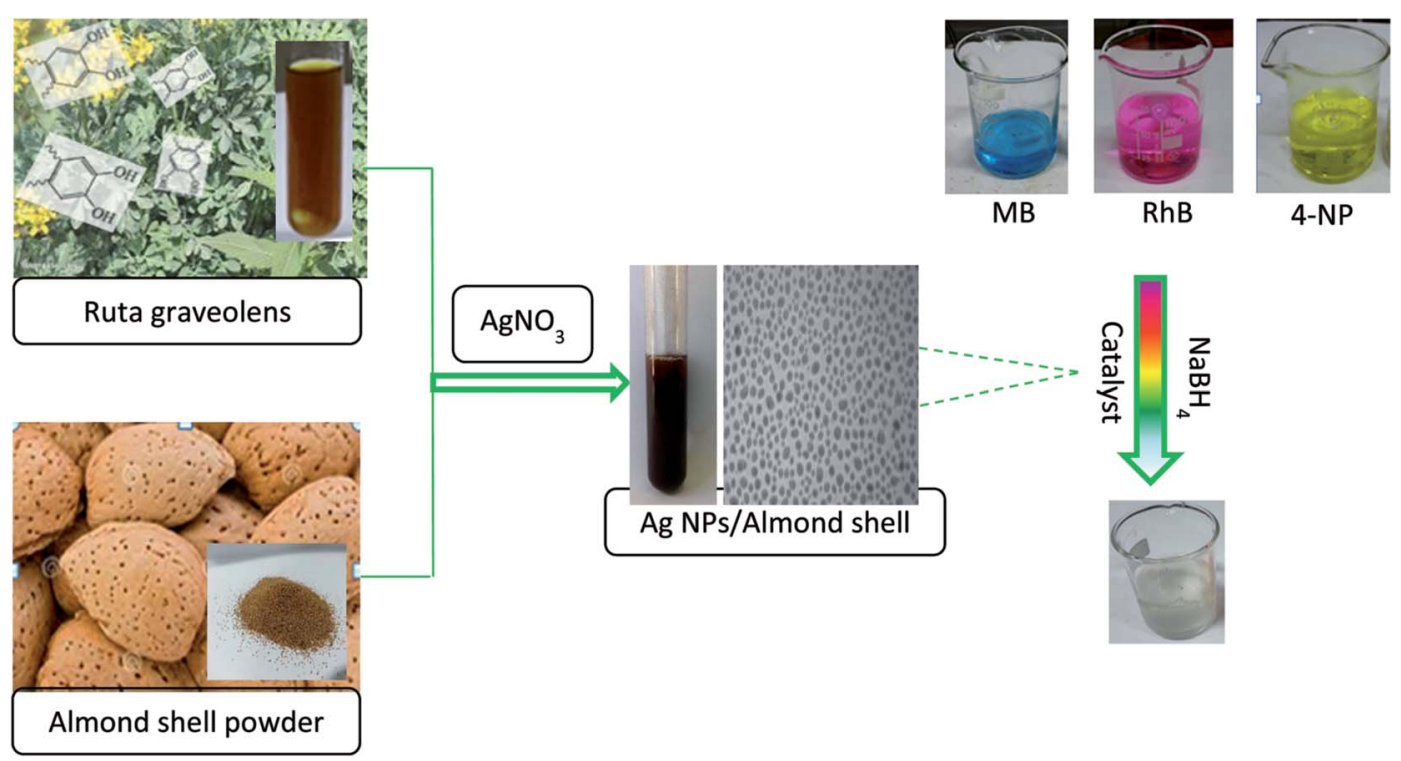

Fig. 3 Flowchart of preparation of Ag/almond shell nanocomposite catalytic reduction and degradation of 4-NP, RhB and MB with Ag/almond shell.<smiles>COc1ccc(C)cc1OCC(C)(C)C</smiles>

Fig. 4 Mechanism involved in the formation of AgNPs. ${ }^{15}$ 
transition localized within coumarin group, whereas the band around $260 \mathrm{~nm}$ is due to the absorbance of ring related to the benzoyl system. These are related to the $\pi \rightarrow \pi^{*}$ transitions and indicate the presence of polyphenolics as antioxidant source for green synthesis of nanoparticles. ${ }^{8,27}$

For the synthesis of AgNPs from silver ion, the phenolic compound present in the extract acting as the reducing agents. The color change (yellowish brown color) during exposure to plant extract clearly indicated the $\mathrm{Ag}(\mathrm{I})$ ion reduction into silver nanoparticles, as shown in the UV-Vis spectra (Fig. 5). Maximum absorbance of green synthesized AgNPs occurred at $430 \mathrm{~nm}$ due to the surface plasmon absorption of nanosized spherical silver particles, as our results showed. ${ }^{21}$

Biomolecules possibly responsible for the capping reduction of AgNPs were identified by FT-IR analysis (Fig. 6a). The peaks at $3500-3400,1680,1460$, and $1250-1050 \mathrm{~cm}^{-1}$ represent free $\mathrm{OH}$ in molecule and $\mathrm{OH}$ group forming hydrogen bonds, carbonyl group $(\mathrm{C}=\mathrm{O})$, stretching $\mathrm{C}=\mathrm{C}$ aromatic ring and $\mathrm{C}-\mathrm{OH}$ stretching vibrations, respectively. The spectrum shows the presence of phenolics in the plant leaf extract due to the presence of the functional groups in the structure of polyphenolics. The reduction of metal ions and formation of nanocomposite is possibly due to the phenolics in the extract. Fig. $6 \mathrm{~b}$ shows the FT-IR spectra of extract and AgNPs. FT-IR spectra of AgNPs are characteristically different in the shape and location of signals

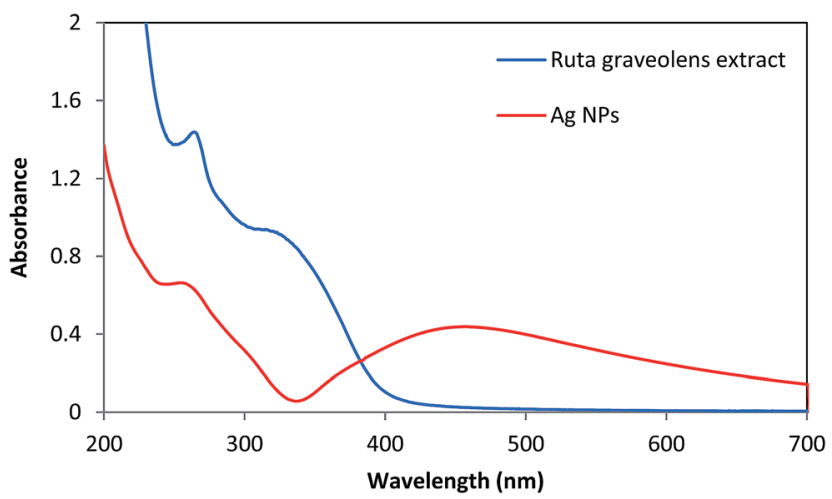

Fig. 5 UV-Vis spectrum of the aqueous extract of the leaves of the Ruta graveolens and green synthesized AgNPs.

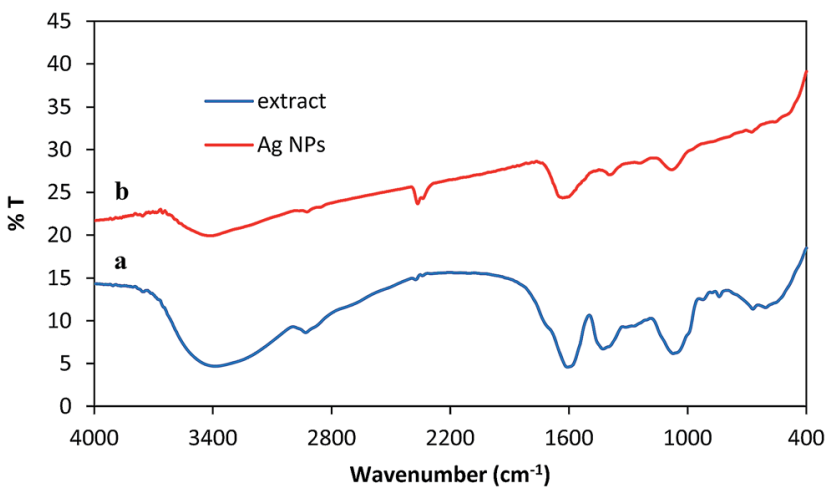

Fig. 6 FT-IR spectrum of the (a) aqueous extract of leaves of Ruta graveolens and (b) synthesized AgNPs.

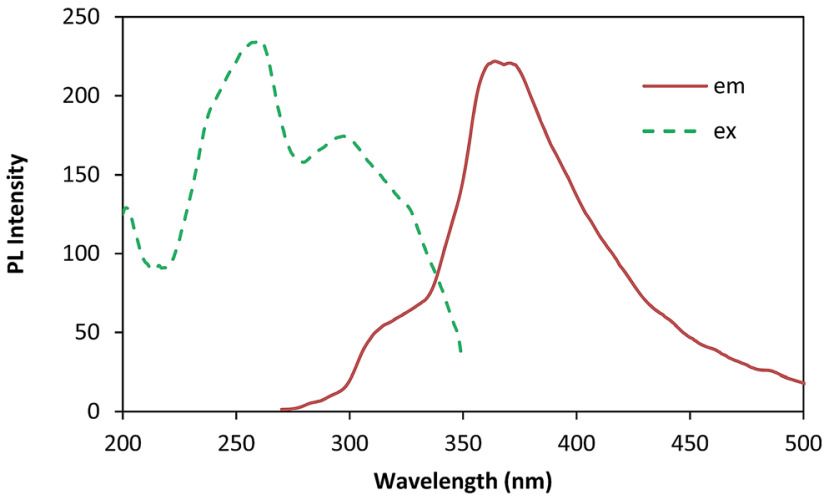

Fig. 7 Photoluminescent spectra with the excitation spectra (dashed lines) and emission spectra (solid lines) of green synthesized AgNPs.

as a result of the interaction between $\mathrm{AgNO}_{3}$ and the phytochemical sites involved in nanoparticle formation (Fig. 6b). Polyphenolics could be possibly adsorbed on the surface of metal nanoparticles by interaction through $\pi$-electrons in the absence of other strong ligating agents. The synthetic AgNPs seem to be stabilized by an organic layer originating from the extract of leaves of Ruta graveolens. Polyphenolics well stabilize these nanoparticles via the interaction of AgNPs with the $\mathrm{OH}$ functional groups.

The photoluminescence (PL) spectra of AgNPs obtained with $260 \mathrm{~nm}$ excitation are shown in Fig. 7. A strong and well defined peak is observed in the PL spectra at around $363 \mathrm{~nm}$. The enhancement of electron density in the polyphenolics compound from leaf extract, adsorbed on the surface of metal nanoparticles probably gives rise to the high photoluminescent intensity. This electron density is the major contributor to photoluminescent emission.

\subsection{Characterization of almond shell and Ag/almond shell nanocomposite}

The FT-IR spectra of almond shells and Ag/almond shell, which are characteristic of cellulose content, are shown in Fig. 8. The presence of hydroxyl group (-OH) stretching is indicated by the broad peaks, observed mainly in raw materials at around 3430 $\mathrm{cm}^{-1}$. The peaks between 2950 and $2850 \mathrm{~cm}^{-1}$ are associated with aliphatic $\mathrm{C}-\mathrm{H}$ deforming vibration. The presence of atmospheric carbon dioxide is indicated by the small band at $2398 \mathrm{~cm}^{-1}$. The bands around $1740 \mathrm{~cm}^{-1}$ are assigned to $\mathrm{C}=\mathrm{O}$ vibration in carbonyl group or the presence of carboxylic bonds. The bands observed at around $1600 \mathrm{~cm}^{-1}$ are due to the aromatic $\mathrm{C}=\mathrm{O}$ ring stretching (possibly $\mathrm{COOH}$ ) or $\mathrm{C}=\mathrm{C}$ stretching of aromatic groups in lignin, implying the presence of lignin. ${ }^{28}$ The broad peak in the $1040-1070 \mathrm{~cm}^{-1}$ region may be due to $\mathrm{C}-\mathrm{OH}$ stretching vibration of alcoholic groups and carboxylic acids. ${ }^{29}$

The expected crystallinity of AgNPs is shown by the powder XRD patterns for the almond shell and Ag/almond shell nanocomposite. The typical broad peak of the carbonaceous structure in the XRD of the almond shell sample is due to the amorphous organic matter (cellulose, hemicelluloses and 


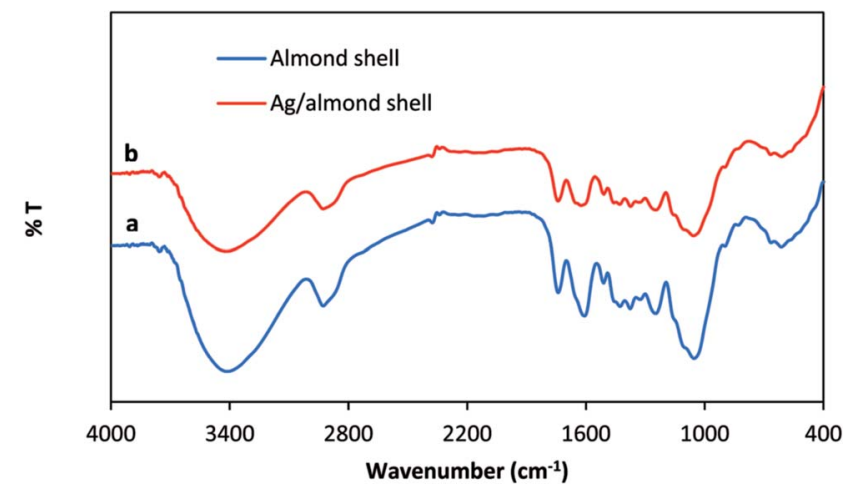

Fig. 8 FT-IR spectrum of the (a) almond shell (b) synthesized Ag/ almond shell nanocomposite.

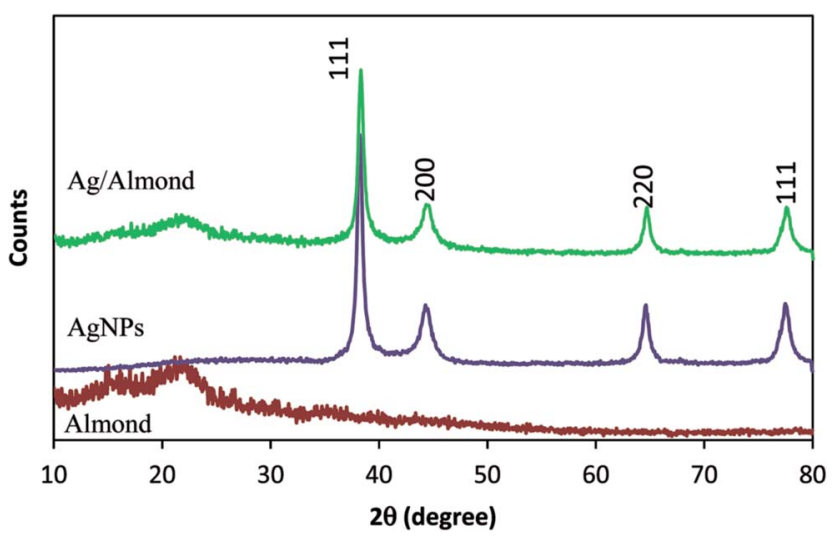

Fig. 9 XRD powder pattern of almond shell, AgNPs Ag/almond shell nanocomposite.

lignin) (Fig. 9). ${ }^{28,30}$ Broad peaks of $2 \theta$ values of 38.2, 44.21, 63.2 and 77.3 degrees, assigned to the corresponding 111, 200, 220 and 311 indices of the face centered cubic (FCC) lattice in accordance with Ag immobilization, are shown in Fig. 9. ${ }^{19}$ The pattern is very clean, showing no sign of impurities such as silver oxide $\left(\mathrm{Ag}_{2} \mathrm{O}\right)$.

The crystalline size is determined by applying DebyeScherrer formula: ${ }^{31}$

$$
D=k \lambda / \beta \cos \theta
$$

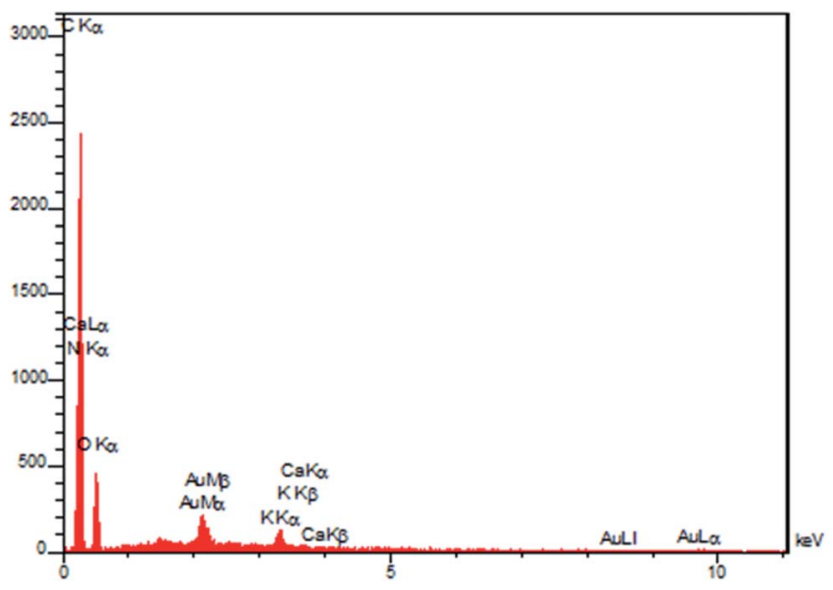

Fig. 11 EDS spectrum of almond shell.

where $D$ is the crystalline size, $\beta$ is the full width half maximum (FWHM) of the $2 \theta$ peak, $k$ is the shape of particle factors (it equals to 0.89), $\theta$ and $l$ are the incident of angle and wavelength of the X-rays, respectively. The average crystalline size of AgNPs associated with the all diffraction peaks was estimated about $15.20 \mathrm{~nm}$. This result is in close agreement with the particle size determined by TEM.

FE-SEM was used in the characterization of the morphology of almond shell (Fig. 10). Almond shell was shown to have a sheet like structure. In addition, a series of $1 \mu \mathrm{m}$ pores are observed on the almond surface. The interesting point is that the porous structure is similar to the structure of almond shell and may possess the catalyst porosity for dye absorption and higher efficiency.

EDS spectroscopy was used to analyze the elemental composition of seashell, confirming the presence of $\mathrm{C}, \mathrm{O}, \mathrm{N}$ and Ca in the almond shell (Fig. 11).

Furthermore, the presence of AgNPs has been verified by EDS analysis (Fig. 13). The amount of Ag incorporated into the Ag/almond shell nanocomposite, determined by EDS, was $7.3 \mathrm{wt} \%$.

The synthesized AgNPs have been immobilized on the almond shell surface, as shown by FE-SEM of Ag/almond shell (Fig. 12). The presence of $\mathrm{Ag}$ was also verified by the elemental
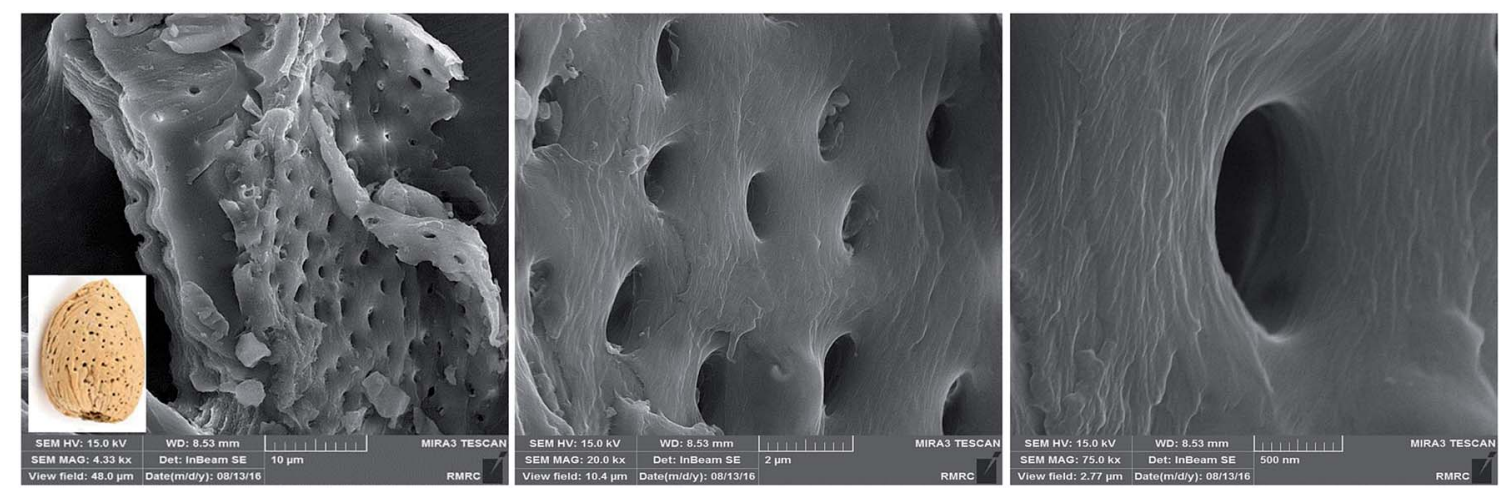

Fig. 10 SEM images of almond shell. 

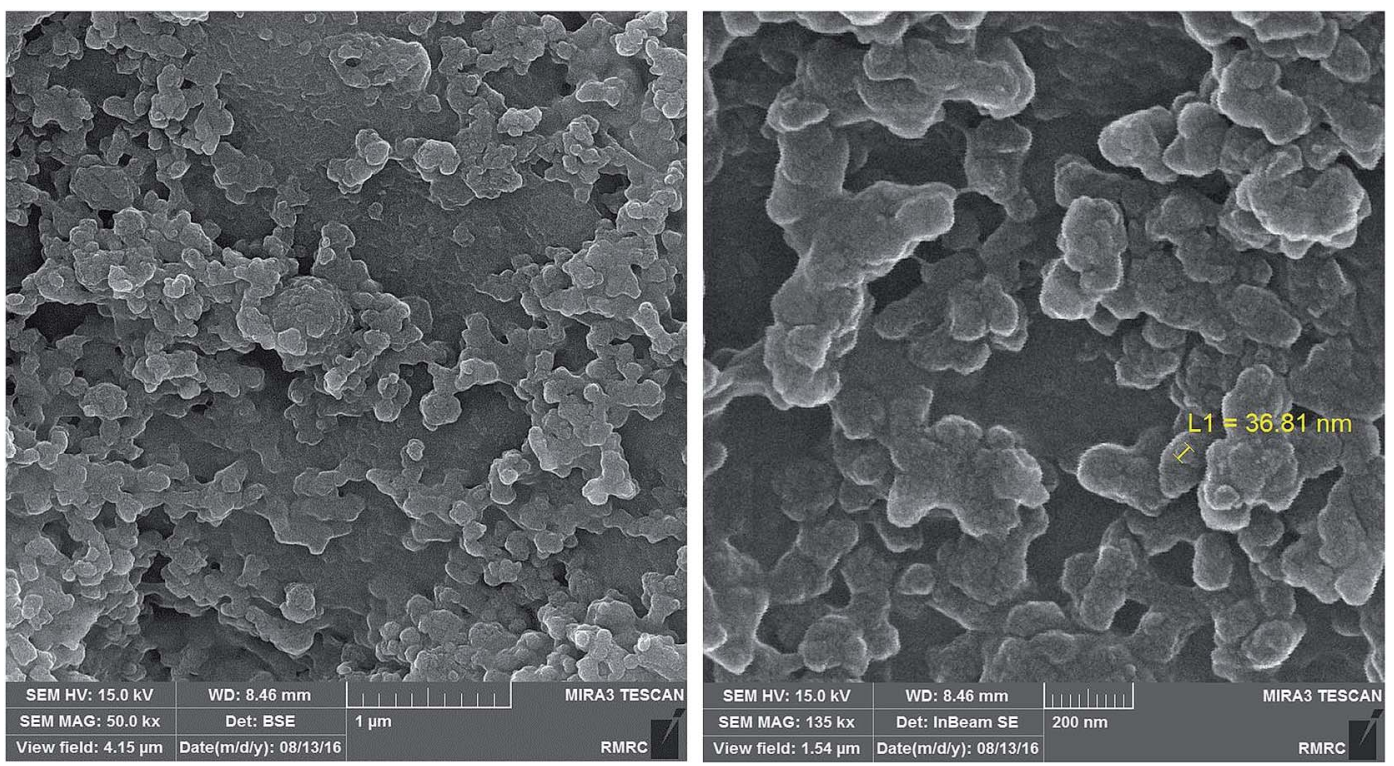

Fig. 12 FE-SEM images of Ag/almond shell.

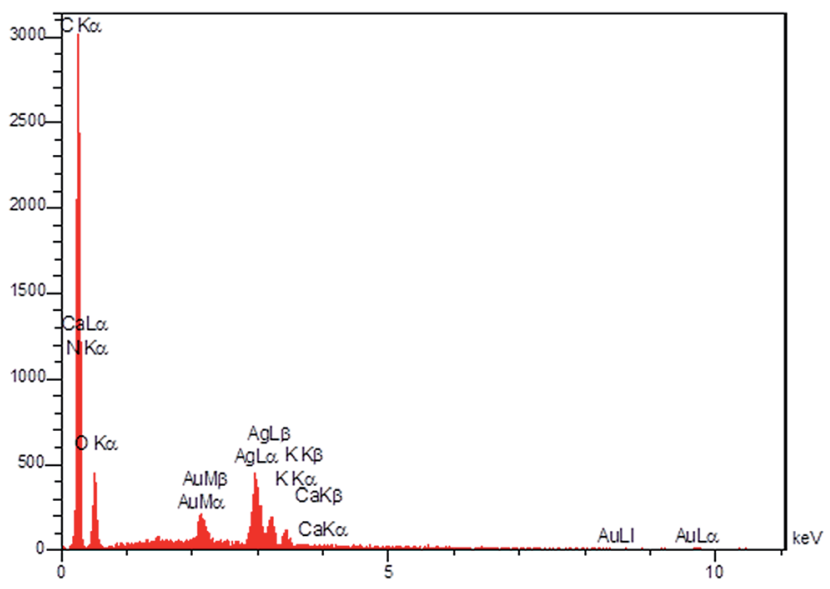

Fig. 13 EDS spectrum of Ag/almond shell.

mapping images (Fig. 14). TEM was used to examine the size and shape of the AgNPs on the surfaces of almond shell (Fig. 15). The particle size of spherical silver NPs on the surface of support is observed to be $10-15 \mathrm{~nm}$. It is observed that size of AgNPs correspond with the calculated size by Scherrer equation.

AgNPs have thus been successfully synthesized and immobilized on the almond shell surface based on the results of XRD, SEM and TEM for the Ag/almond shell nanocomposite.

\subsection{Degradation of 4-NP using green synthesized AgNPs/ almond shell}

The reduction of aqueous solutions of 4-NP in the presence of $\mathrm{NaBH}_{4}$ at ambient temperature was used to evaluate the catalytic activity of the Ag/almond shell nanocomposite (Scheme 1). UV-visible spectroscopy measurements at regular time intervals were used to monitor the progress of the reaction. 4-NP has a maximum absorption at $317 \mathrm{~nm}$ in aqueous medium (Fig. 16).
In the absence of the catalyst, the peak at $317 \mathrm{~nm}$ does not change even after a couple of days. $2.5 \mathrm{mM}$ solution of $4-\mathrm{NP}$ $(25.0 \mathrm{~mL}$ ) was mixed with a freshly prepared solution of $0.25 \mathrm{M}$ aqueous $\mathrm{NaBH}_{4}(25.0 \mathrm{~mL})$ and $\mathrm{Ag} /$ almond shell nanocomposite $(5.0 \mathrm{mg})$ at ambient temperature to evaluate the catalytic activity of the Ag/almond shell nanocomposite. Upon addition of $\mathrm{NaBH}_{4}$ into 4-NP solution, the light yellow color of the solution changed to intense yellow, showing an absorption peak at about $405 \mathrm{~nm}$ due to the formation of 4-nitrophenolate ions under alkaline conditions, which did not change as time passed. When Ag/almond shell nanocomposite was added into the reaction mixture, the peak at $400 \mathrm{~nm}$ decreased and a new peak corresponding to the formation of 4-aminophenol (4-AP) appeared at about $300 \mathrm{~nm}$ (Fig. 16). The whole peak at $400 \mathrm{~nm}$ disappeared after about $4.5 \mathrm{~min}$ and the yellow color of the solution changed to white, indicating the conversion of 4-NP to 4-AP. Therefore, pure AgNPs can catalyze 4-NP reduction to 4-AP, but at slower reaction rates than Ag/almond shell nanocomposite. A longer reaction time of 15 minutes was required for complete reduction of 4-NP to 4-AP by $\mathrm{NaBH}_{4}$ using pure AgNPs (Table 1). Thus, Ag/almond shell nanocomposite has a higher catalytic activity in the reduction of 4-NP to 4-AP compared with AgNPs alone. This can be attributed to the possibility of the almond shell support playing an active part in the catalysis, leading to a synergistic effect. ${ }^{8}$

\subsection{Degradation of RhB and MB using green synthesized AgNPs/almond shell}

Significant pollution is caused by dyes and other dyestuffs, which are the major effluents from the textile industry. ${ }^{32,33}$ Most dyes are not biodegradable. Thus, the development of an environmentally friendly method for reduction of these compounds in aqueous media is highly desirable. The reductions of RhB and $\mathrm{MB}$ by $\mathrm{NaBH}_{4}$ have been chosen as model reactions to study 

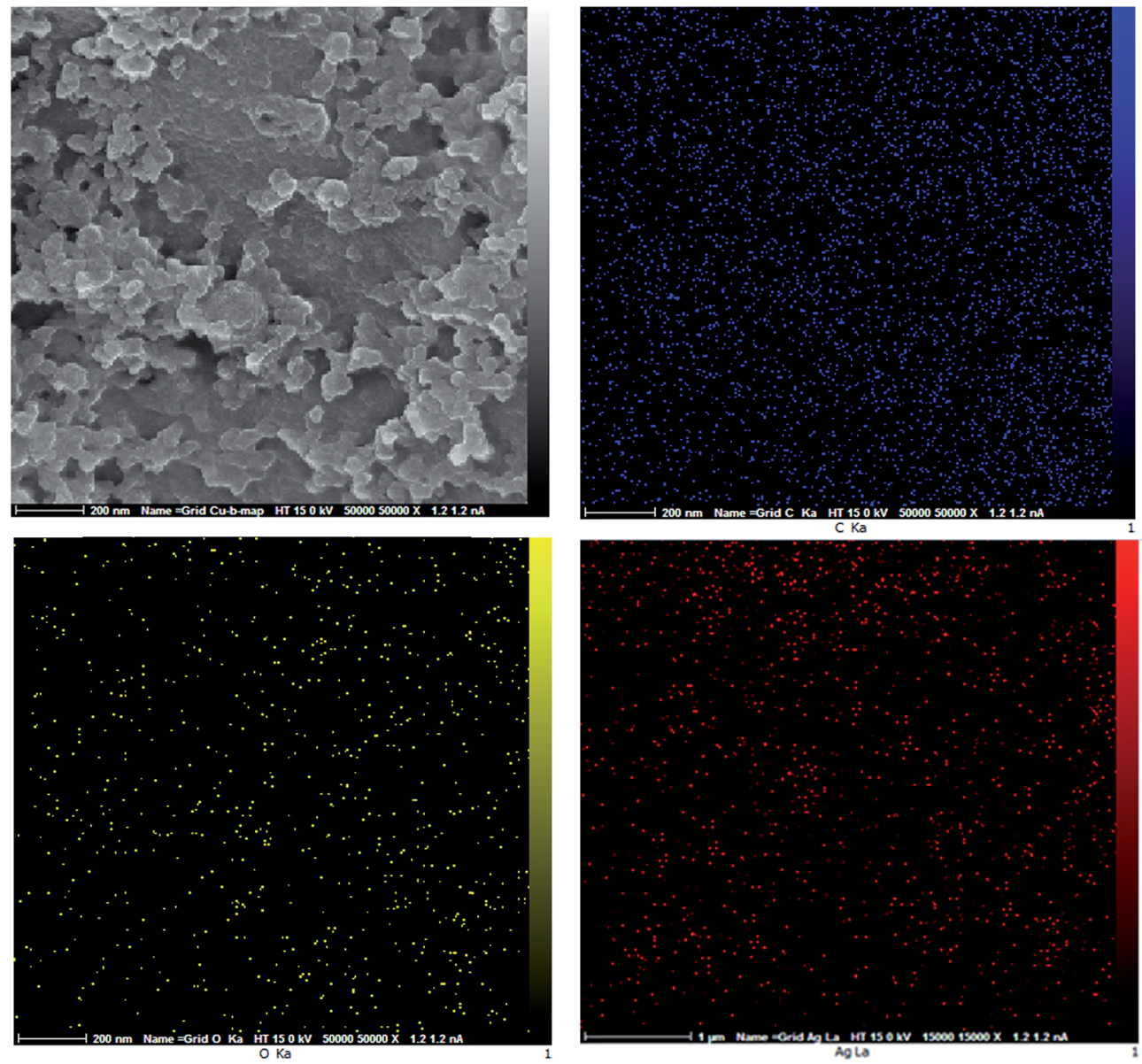

Fig. 14 Elemental mappings for Ag/almond shell nanocomposite; (blue) C, (yellow) O, Ca, and (red) Ag.
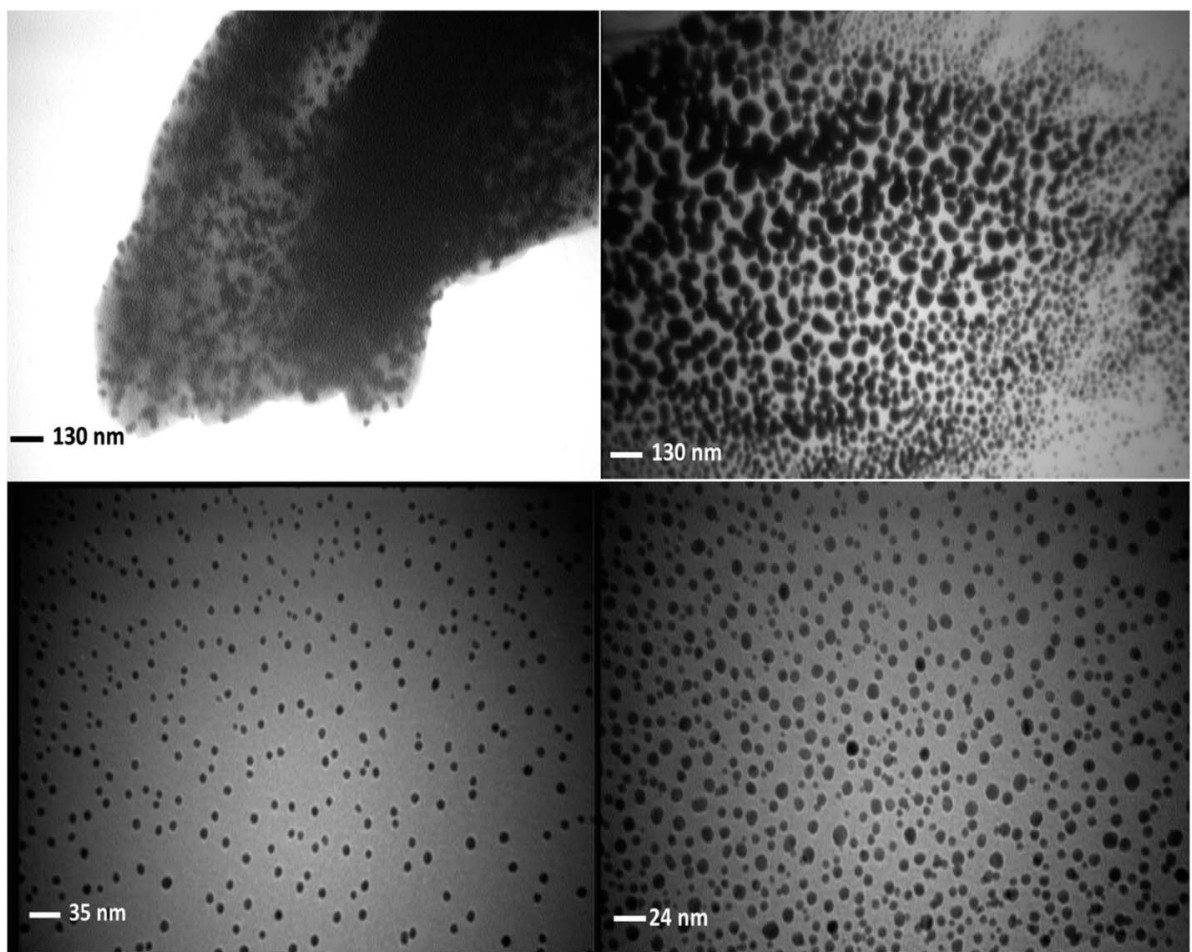

Fig. 15 Typical TEM images of AgNPs/almond shell. 
<smiles>Nc1ccc(O)cc1CCc1cc([N+](=O)[O-])ccc1[N+](=O)[O-]</smiles>

Scheme 1 Mechanism of the catalytic reduction and degradation of 4-NP with Ag/almond shell nanocomposite at room temperature.

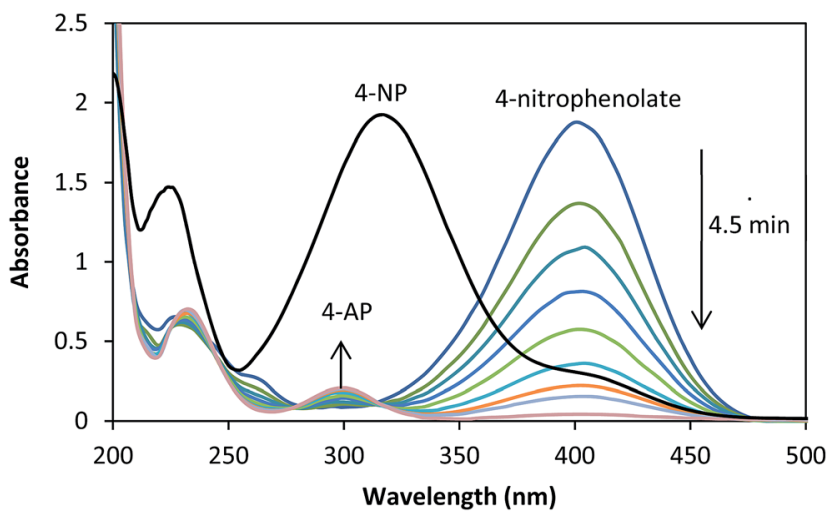

Fig. 16 UV-visible spectra for catalytic reduction of 4-NP to 4-AP at several interval, conditions: $[4-\mathrm{NP}]=2.5 \times 10^{-3} \mathrm{M},\left[\mathrm{NaBH}_{4}\right]=0.25 \mathrm{M}$, Ag/almond shell $=5.0 \mathrm{mg}$.

Table 1 Completion time for the reduction of $25 \mathrm{~mL}$ of $\mathrm{RhB}$ and $\mathrm{MB}$ dyes $(10 \mathrm{ppm})$ in the presence of $25 \mathrm{~mL}$ of $\mathrm{NaBH}_{4}\left(2.6 \times 10^{-3} \mathrm{M}\right)$ and 4-NP (2.5 mM) in the presence of $25 \mathrm{~mL}$ of $\mathrm{NaBH}_{4}(0.25 \mathrm{M})$, using $5 \mathrm{mg}$ of AgNPs, almond shell and $\mathrm{Ag} / \mathrm{almond}$ shell

\begin{tabular}{llcr}
\hline & \multicolumn{2}{c}{ Time (min) } & \\
\cline { 2 - 4 } Catalyst & 4 -NP & RhB & MB \\
\hline Ag/almond shell & 4.5 & 2 & $10 \mathrm{~s}$ \\
AgNPs & 15 & 9 & 5 \\
Almond shell & $45^{a}$ & $45^{a}$ & $45^{a}$
\end{tabular}

${ }^{a}$ Not completed.

the performance of the synthesized Ag/almond shell nanocomposite. The sequences of the steps in this reaction are: adsorption of $\mathrm{NaBH}_{4}$ onto the surface of catalyst to form metal hydride, adsorption of $\mathrm{MB}(\mathrm{RhB})$ onto the surface of catalyst, and reduction and desorption of $\mathrm{MB}(\mathrm{RhB})$ to create a free space for the reaction to continue. The decrease of absorbance at $\lambda_{\max }$ $\left(\lambda_{\max }(\mathrm{RhB})=550 \mathrm{~nm}\right.$ and $\left.\lambda_{\max }(\mathrm{MB})=663 \mathrm{~nm}\right)$ with time was used to spectrophotometrically monitor the reduction of $\mathrm{RhB}$ and $\mathrm{MB}$ by $\mathrm{NaBH}_{4}$ (Fig. 17). RhB and MB catalytic reduction by $\mathrm{NaBH}_{4}$ proceeded successfully, as expected (Table 1). The effects of the amount of the catalyst and $\mathrm{NaBH}_{4}$ were determined for reduction reaction. No product was obtained in the absence of the catalyst. Thus, indicating that the catalytic reduction occurs at the surface of catalyst. In the absence of catalyst, no significant color change was observed within the reaction time and the peak remained unaltered for a long duration. On the other
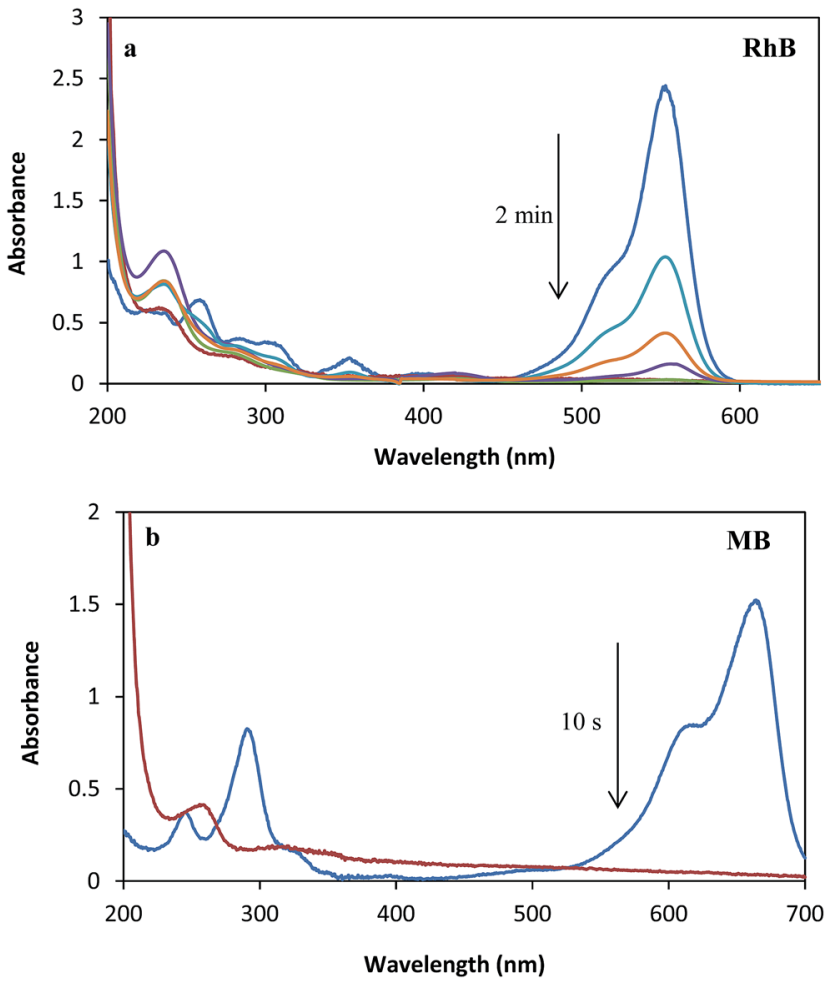

Fig. 17 UV-Vis spectra of reduction of (a) RhB $\left(2.1 \times 10^{-5} \mathrm{M}\right)$ and (b) $\mathrm{MB}\left(3.1 \times 10^{-5} \mathrm{M}\right)$ with $\left[\mathrm{NaBH}_{4}\right]=2.6 \times 10^{-3} \mathrm{M}$ in presence of $\mathrm{Ag} /$ almond shell nanocomposite $=5.0 \mathrm{mg}$.

hand, in the absence of $\mathrm{NaBH}_{4}$, no reduction occurred in the presence of catalyst. Also, no significant color change was observed in the presence of catalyst and in the absence of $\mathrm{NaBH}_{4}$. Thus, the reduction reaction was carried out in the presence of catalyst and $\mathrm{NaBH}_{4}$.

Table 2 Comparison of various catalysts in the reduction of 4-NP, $\mathrm{RhB}$ and $\mathrm{MB}$ dyes with $\mathrm{NaBH}_{4}$

\begin{tabular}{|c|c|c|c|}
\hline Substrate & Catalyst & Time & Ref. \\
\hline \multirow[t]{8}{*}{ 4-NP } & $\mathrm{Ag} / \mathrm{KCC}-1$ & $8.5 \mathrm{~min}$ & 34 \\
\hline & $\mathrm{Cu}$ microspheres & $18 \mathrm{~min}$ & 35 \\
\hline & Ni NPs & $16 \mathrm{~min}$ & 36 \\
\hline & Pd-FG & $12 \min$ & 37 \\
\hline & Ni-PVAm/SBA-15 & $85 \min$ & 38 \\
\hline & $\mathrm{Fe}_{3} \mathrm{O}_{4} @ \mathrm{SN}-\mathrm{Ag}$ & $10 \mathrm{~min}$ & 39 \\
\hline & Ag/GO-Dopa & $8 \mathrm{~min}$ & 40 \\
\hline & $\mathrm{Ag} /$ almond shell & $4 \mathrm{~min}$ & This work \\
\hline \multirow[t]{5}{*}{ RhB } & $\mathrm{Fe}_{3} \mathrm{O}_{4} / \mathrm{Ag}$ & $15 \mathrm{~min}$ & 41 \\
\hline & $\mathrm{PS} / \mathrm{Ag}$ & $10 \min$ & 42 \\
\hline & $\mathrm{Ag} / \mathrm{HLaNb}_{2} \mathrm{O}_{7}$ & $47 \mathrm{~min}$ & 3 \\
\hline & $\begin{array}{l}\mathrm{Ag} / \text { zeolite } \\
\text { nanocomposite }\end{array}$ & $7.5 \mathrm{~min}$ & 19 \\
\hline & $\mathrm{Ag} /$ almond shell & $2 \mathrm{~min}$ & This work \\
\hline \multirow[t]{6}{*}{ MB } & $\begin{array}{l}\mathrm{Ag} / \text { zeolite } \\
\text { nanocomposite }\end{array}$ & $50 \mathrm{~s}$ & 7 \\
\hline & AgNPs on silica spheres & $7.5 \mathrm{~min}$ & 3 \\
\hline & SiNWAs-Cu & $10 \mathrm{~min}$ & 43 \\
\hline & $\mathrm{Au} @ \mathrm{TiO}_{2}$ & $12 \mathrm{~min}$ & 44 \\
\hline & Ru nanocrystals & $9 \min$ & 45 \\
\hline & Ag/almond shell & $31 \mathrm{~s}$ & This work \\
\hline
\end{tabular}


The applicability and efficiency of the synthetic catalysts was evaluated by comparison of the results with those of the recently reported methods for the reduction of variety of dyes by $\mathrm{NaBH}_{4}$ (Table 2). The proposed method is the shortest method for the reduction of organic dyes reported so far.

\subsection{Catalyst recyclability}

The recovery and recyclability of the AgNPs/almond shell was also studied in 4-NP reduction. Upon completion of the reaction, monitored by UV-Vis spectroscopy, the catalyst was removed from the reaction mixture by mild centrifugation, washed several times with distilled water, dried and used in the next reactions. The catalyst was observed to function well up to five catalytic runs with $100 \%$ reduction of $4-\mathrm{NP}$, demonstrating the high stability and catalytic activity of $\mathrm{Ag} / \mathrm{almond}$ shell under the operating conditions. The recyclability of the AgNPs/almond shell was also studied in the reduction of RhB and MB under the reaction conditions. The results of recycling experiments showed that the catalyst could be recycled at least five times for $100 \%$ reduction of $\mathrm{RhB}$ and $\mathrm{MB}$.

\section{Conclusions}

$\mathrm{Ag} /$ almond shell nanocomposite has been synthesized via a green method using the extract of leaves of Ruta graveolens. Phenolic acid and flavonoids in the Ruta graveolens leaf extract, both of which act as reducing and stabilizing agents, probably facilitate the formation of the Ag/almond shell nanocomposite by $\mathrm{Ag}(\mathrm{I})$ reduction. Strong catalytic activity is shown by $\mathrm{Ag} /$ almond shell nanocomposite in the base and ligand free oxidative hydroxylation of phenylboronic acid to phenol and the reduction of $4-\mathrm{NP}, \mathrm{RhB}$ and $\mathrm{MB}$ at ambient temperature. This simple and versatile protocol has been used in the synthesis of phenol in high yields. The catalyst could be recycled several times with no loss of catalytic activity.

\section{Acknowledgements}

The author is thankful to Iran National Science Foundation (INSF) for supporting this project numbered 95823609.

\section{Notes and references}

1 P. K. Jain, X. Huang, I. H. El-Sayed and M. A. El-Sayed, Acc. Chem. Res., 2008, 41, 1578-1586.

2 J. M. Campelo, D. Luna, R. Luque, J. M. Marinas and A. A. Romero, ChemSusChem, 2009, 2, 18-45.

3 Z.-J. Jiang, C.-Y. Liu and L.-W. Sun, J. Phys. Chem. B, 2005, 109, 1730-1735.

4 N. Pradhan, A. Pal and T. Pal, Colloids Surf., A, 2002, 196, 247-257.

5 E. Qayyum, V. A. Castillo, K. Warrington, M. A. Barakat and J. N. Kuhn, Catal. Commun., 2012, 28, 128-133.

6 A. Keshavaraja, X. She and M. Flytzani-Stephanopoulos, Appl. Catal., B, 2000, 27, L1-L9.
7 A. Hatamifard, M. Nasrollahzadeh and S. M. Sajadi, New J. Chem., 2016, 40, 2501-2513.

8 M. Atarod, M. Nasrollahzadeh and S. Mohammad Sajadi, J. Colloid Interface Sci., 2016, 462, 272-279.

9 H. Li, Y. Zhang, G. Chang, S. Liu, J. Tian, Y. Luo, A. M. Asiri, A. O. Al-Youbi and X. Sun, ChemPlusChem, 2012, 77, 545-550.

10 P. Zhang, C. Shao, Z. Zhang, M. Zhang, J. Mu, Z. Guo and Y. Liu, Nanoscale, 2011, 3, 3357-3363.

11 S. P. Dubey, M. Lahtinen and M. Sillanpää, Process Biochem., 2010, 45, 1065-1071.

12 A. B. S. Sastry, R. B. Karthik Aamanchi, C. Sree Rama Linga Prasad and B. S. Murty, Environ. Chem. Lett., 2013, 11, 183187.

13 S. Basu, P. Maji and J. Ganguly, Appl. Nanosci., 2016, 6, 1-5.

14 S. Ahmed, M. Ahmad, B. L. Swami and S. Ikram, J. Adv. Res., 2016, 7, 17-28.

15 T. J. I. Edison and M. G. Sethuraman, Process Biochem., 2012, 47, 1351-1357.

16 A. Singh, D. Jain, M. Upadhyay, N. Khandelwal and H. Verma, Digest Journal of Nanomaterials and Biostructures, 2010, 5, 483-489.

17 S. Li, Y. Shen, A. Xie, X. Yu, L. Qiu, L. Zhang and Q. Zhang, Green Chem., 2007, 9, 852-858.

18 H. Bar, D. K. Bhui, G. P. Sahoo, P. Sarkar, S. Pyne and A. Misra, Colloids Surf., A, 2009, 348, 212-216.

19 A. Hatamifard, M. Nasrollahzadeh and S. M. Sajadi, New J. Chem., 2016, 40, 2501-2513.

20 A. Rostami-Vartooni, M. Nasrollahzadeh and M. Alizadeh, J. Colloid Interface Sci., 2016, 470, 268-275.

21 T. N. J. I. Edison, Y. R. Lee and M. G. Sethuraman, Spectrochim. Acta, Part A, 2016, 161, 122-129.

22 H. Ekiert, A. Szewczyk and A. Kuś, Die Pharmazie, 2009, 64, 692-694.

23 A. L. Dawidowicz, K. Bernacik and R. Typek, Food Analytical Methods, 2016, 9, 213-224.

24 J. M. Martínez, J. Reguant, M. Á. Montero, D. Montané, J. Salvadó and X. Farriol, Ind. Eng. Chem. Res., 1997, 36, 688-696.

25 H. Pirayesh and A. Khazaeian, Composites, Part B, 2012, 43, 1475-1479.

26 M. A. Abedini Najafabadi, S. N. Khorasani and J. M. Esfahani, Polym. Polym. Compos., 2014, 22, 409-416.

27 M. Kamiński, R. Kartanowicz, M. M. Kamiński, A. Królicka, M. Sidwa-Gorycka, E. Łojkowska and W. Gorzeń, J. Sep. Sci., 2003, 26, 1287-1291.

28 M. Konsolakis, N. Kaklidis, G. E. Marnellos, D. Zaharaki and K. Komnitsas, RSC Adv., 2015, 5, 73399-73409.

29 S. Hashemian and J. Shayegan, Orient. J. Chem., 2014, 30, 2091-2098.

30 B. Peters, Fuel Process. Technol., 2011, 92, 1993-1998.

31 A. L. Patterson, Phys. Rev., 1939, 56, 978-982.

32 M. Bordbar, B. Khodadadi, N. Mollatayefeh and A. YeganehFaal, J. Appl. Chem., 2013, 8, 1-5.

33 B. Khodadadi, M. Bordbar and A. Yeganeh-Faal, J. Sol-Gel Sci. Technol., 2016, 77, 521-527.

34 Z. Dong, X. Le, X. Li, W. Zhang, C. Dong and J. Ma, Appl. Catal., B, 2014, 158-159, 129-135. 
35 E. S. Abdel-Halim, M. H. El-Rafie and S. S. Al-Deyab, Carbohydr. Polym., 2011, 85, 692-697.

36 Z. Jiang, J. Xie, D. Jiang, X. Wei and M. Chen, CrystEngComm, 2013, 15, 560-569.

37 Z. Wang, C. Xu, G. Gao and X. Li, RSC Adv., 2014, 4, 1364413651.

38 R. J. Kalbasi, A. A. Nourbakhsh and F. Babaknezhad, Catal. Commun., 2011, 12, 955-960.

39 Z.-Z. Wang, S.-R. Zhai, F. Zhang, Z.-Y. Xiao, B. Zhai and Q.-D. An, J. Sol-Gel Sci. Technol., 2015, 73, 299-305.
40 E. K. Jeon, E. Seo, E. Lee, W. Lee, M.-K. Um and B.-S. Kim, Chem. Commun., 2013, 49, 3392-3394.

41 L. Ai, C. Zeng and Q. Wang, Catal. Commun., 2011, 14, 68-73. 42 Z. Deng, H. Zhu, B. Peng, H. Chen, Y. Sun, X. Gang, P. Jin and J. Wang, ACS Appl. Mater. Interfaces, 2012, 4, 5625-5632.

43 X. Yang, H. Zhong, Y. Zhu, H. Jiang, J. Shen, J. Huang and C. Li, J. Mater. Chem. A, 2014, 2, 9040-9047.

44 M. M. Khan, J. Lee and M. H. Cho, J. Ind. Eng. Chem., 2014, 20, 1584-1590.

45 B. K. Ghosh, S. Hazra, B. Naik and N. N. Ghosh, J. Nanosci. Nanotechnol., 2015, 15, 6516-6523. 